\title{
MASS AND ENERGY BALANCE LAWS DERIVED FROM HIGH-FIELD LIMITS OF THERMOSTATTED BOLTZMANN EQUATIONS*
}

\author{
P. DEGOND ${ }^{\dagger}$ AND B. WENNBERG ${ }^{\ddagger}$
}

\begin{abstract}
We derive coupled mass and energy balance laws from a High-Field limit of thermostatted Boltzmann equations. The starting point is a Boltzmann equation for elastic collisions subjected to a large force field. By adding a thermostat correction, it is possible to expand the solutions about a High-Field equilibrium obtained when balancing the thermostatted field drift operator with the elastic collision operator. To this aim, a hydrodynamic type scaling of the thermostatted Boltzmann equation is used, considering that the leading 'collision operator' actually consists of the combination of the thermostatted field operator and of the elastic collision operator. At leading order in the Knudsen number, the resulting model consist of coupled nonlinear first order partial differential equations. We investigate two cases. The first one is based on a one-dimensional BGK-type operator. The second one is three dimensional and concerns a Fokker-Planck collision operator. In both cases, we show that the resulting models are hyperbolic, thereby indicating that they might be appropriate for a use in physically realistic situations.
\end{abstract}

Key words. Boltzmann equation, Fokker-Planck equation, thermostat, hydrodynamic limit, High-Field limit, hyperbolic balance laws, mass and energy transfer

AMS subject classifications. $\quad 82 \mathrm{C} 70,82 \mathrm{C} 40,82 \mathrm{C} 21,82 \mathrm{~B} 40,82 \mathrm{~B} 21,35 \mathrm{~J} 05,76 \mathrm{M} 28$

\section{Introduction}

In semiconductor or plasma physics, much use is made of mass and energy transfer models which sometimes bear the name (mostly in the semiconductor community) of Energy-Transport models [1, 2, 3, 14, 20, 21, 23, 34, 40, 48, 49] (see also the review articles [18], [19] and references therein). They consist of coupled convection-diffusion equations for the particle and energy densities of each species of particles. They are valid in situations where the particles (e.g. the electrons and holes in semiconductors, the electrons and ions in plasmas) evolve under the combined influence of an external or mean-field force (e.g. the electrostatic or electromagnetic force) and collisions with a background (e.g. impurities and phonons in semiconductors, neutral molecules in plasmas).

During a collision event, particles exchange momentum and energy with the background. The relative magnitude of the collision processes compared with the other physical phenomena such as the external or mean-field force is measured by the inverse of the Knudsen number where the Knudsen number represents the ratio of the collision mean free path to the typical length scales of the system. If the collisions are strong, or in other words, if the Knudsen number is small, a quick relaxation of the particle distribution towards a state of Local Thermodynamical Equilibrium (LTE) occurs. In the present case, the LTE is characterized by the same mean velocity and temperature as the background. Usually, the background is at rest and the mean velocity is equal to zero. Then, the LTE only depends on a single free parameter, the chemical potential (or equivalently the dual extensive variable, namely the density),

*Received: September 7, 2006; accepted (in revised version): March 14, 2007. Communicated by Anton Arnold.

${ }^{\dagger}$ MIP, UMR 5640 (CNRS-UPS-INSA), Université Paul Sabatier, 118, route de Narbonne, 31062 Toulouse cedex, France (degond@mip.ups-tlse.fr).

${ }^{\ddagger}$ Department of Mathematical Sciences, Chalmers University of Technology. Department of Mathematical Sciences, Gteborg University, SE-41296 Gteborg, Sweden (wennberg@math.chalmers.se). 
which evolves under a single convection-diffusion equation. This yields the so-called drift-diffusion model which has been widely used in the semiconductor or plasma literature for decades [30], [41], [44], [50].

However, in some situations far from equilibrium, the energy exchanges between the particles and the background are much slower than the momentum exchanges, and for a long period of time, an intermediate equilibrium persists, in which the particle temperature is different from the background temperature. In semiconductors, energy exchanges occur by quanta equal to the phonon energy $\hbar \omega_{0}$ and in sub-microelectronic devices, the typical particle energy is much larger. Therefore, the relative energy exchanges between electrons and phonons during one single collision event are very small. In plasmas, the small mass ratio between the electrons and the neutrals or ions also makes the energy exchange processes extremely inefficient.

In such situations, it is legitimate to say that the LTE is characterized by two parameters instead of one, the chemical potential (or the density) and the temperature (or the energy density). These two quantities now evolve by means of two coupled diffusion equations. This yields the so-called Energy-Transport model. Its derivation from the Boltzmann equation by means of a Hilbert perturbation expansion has been outlined in [23] in the plasma context and by [3] and [2] in the semiconductor context (see also the reviews [18], [19]). In these references, the entropic structure of the Energy-Transport model has been emphasized: indeed, the fluxes of the conservative variables (density and energy) are expressed in terms of the gradients of the dual entropic variables (chemical potential and temperature) through a symmetric positivedefinite diffusion matrix. For numerical methods we refer the reader to [21], and for recent applications to [34].

In both the Drift-Diffusion and Energy-Transport models, the LTE (a Maxwellian in the case of Boltzmannian particles, a Fermi-Dirac distribution in the case of Fermions) is a spherically symmetric function of the microscopic particle momenta. However, in the presence of strongly non-equilibrium phenomena, the applied forces (typically the electric or electromagnetic forces in the case of plasmas or semiconductors) strongly modify the shape of the momentum distribution function and generate an anisotropy in the direction of the force field. This indicates that a perturbation expansion about a spherically symmetric LTE is not legitimate. In such regimes, both the Drift-Diffusion and Energy-Transport models must be invalid (although the Energy-Transport model, by assuming a different temperature than the background, is probably less inaccurate). This remark has been the starting point of many studies in which new macroscopic models are sought by expanding the distribution function about "High-Field Equilibria" obtained by balancing the actions of the field and of the collisions.

Such High-Field models have been investigated in [4], [12], [22], [45], [46], [47]. At leading order in the Knudsen number, these models consist of linear or nonlinear hyperbolic equations for the local density. By expanding the distribution function to the next order in the Knudsen number, diffusive corrections can be obtained by means of a Hilbert or Chapman-Enskog technique. In these earlier approaches, it was assumed that the collision operator relaxes to the local Maxwellian with zero mean velocity and fixed background temperature. In other words, it was assumed that the energy exchanges during collisions are of the same order of magnitude as momentum exchanges. In this sense, these High-Field models are a natural extension of the Drift-Diffusion model. Actually, a Small-Field asymptotic of these models (including diffusive corrections) restitutes the Drift-Diffusion model. The existence of a leading 
order distribution function is not an obvious question and is related to integrability conditions on the collision frequency. This question has been investigated in [10], [11], [29], [47].

A natural question is therefore investigating the possibility of High-Field models which would extend the Energy-Transport model. Such a possibility relies on the possibility of making a High-Field limit of a Boltzmann model where the collision operator conserves energy and relaxes the distribution function towards a local Maxwellian with an arbitrary temperature. However, determining the leading order term of the High-Field expansion requires solving an equation for the leading order distribution function where the collision operator is balanced by the field acceleration. Such a problem is obviously ill-posed and actually has no solution at least of finite energy. Indeed, the field brings energy into the physical system which the collisions, being elastic, are unable to dissipate. Actually, it is easily shown that a space-homogeneous distribution function which evolves under the combined actions of the field and of an elastic operator does not converge for large times towards a finite energy distribution function. This problem has prevented the development of High-Field counterparts of the Energy-Transport model for some time.

The present paper reports on the first attempt (to our knowledge) to use a thermostatted field operator in order to overcome this problem.

The notion of thermostats in the sense used here has its origin in molecular dynamics and simulation of non-equilibrium phenomena, with the works of e.g. D. J. Evans, W. G. Hoover, G. P. Morriss and others in the beginning of the eighties (see $[25,26,35]$ and some of the available textbooks $([37,36,28])$. The basic problem is to study (mechanical) systems under the influence of a steady external field. To obtain a stationary state, the energy supplied by the external field must be absorbed by some means, and the so-called Gaussian iso-kinetic thermostat achieves this by adding a (non-holonomic) constraint force that forces the system to remain on a submanifold of constant kinetic energy. Similarly an iso-energetic thermostat constrains the system to conserve energy. The word Gaussian refers to the link with Gauss' principle of least constraint for non-holonomic constraints in mechanical systems.

While the Gaussian thermostats correspond to a micro-canonical ensemble, the Nos-Hoover thermostat aims at simulating the canonical ensemble. There the original Hamiltonian system is supplemented with an additional canonical pair of variables, which model the contact with a reservoir (see [43, 27]).

There are many different forms of both the Gaussian thermostats and the NosHoover thermostats. Some of the applications and qualities of the different versions are described in [38] and [42].

Among the rather few rigorous results concerning thermostatted dynamics, we mention the work by Chernov et al [15, 16], which concerns the Lorentz gas subject to a (small) force field and a Gaussian iso-kinetic thermostat. The authors prove, among other things, that if the force field is sufficiently small, there is a unique stationary measure for the evolution, and that Ohm's law holds. Careful numerical simulations indicate that the same is true for stronger force fields, but that the results depend in an irregular way on the strength of the field $[6,7]$.

The thermostats as described here deal with the evolution of a finite number of particles under the influence of a thermostatted force field. In kinetic theory, one is concerned with the evolution of a phase space density of particles. The Boltzmann equation can be derived as the limit of infinitely many particles that obey the classical laws of mechanics. The Boltzmann equation for a system of particles that are subject 
to a thermostatted force field has been studied by Wondmagegne $[53,52,51,5]$. A formal derivation of a Boltzmann equation for a thermostatted system is carried out, and rigorous results on non-equilibrium stationary states are obtained for Kac's toy model of the Boltzmann equation [39].

Here, the usage of thermostats will be slightly different. They will help us in determining the leading order term in the High-Field expansion of the kinetic equation. However, the overall energy budget of the problem will not be modified, and actually, the energy increase due to the field operator will be duely accounted for in the limit model. To this aim, we add and subtract the thermostat correction, leading to corrections and counter-correction terms in the kinetic equation whose respective contributions exactly cancel each other. However, the thermostat correction is grouped with the field and collision operators in the leading order term of the Hilbert expansion, while the counter-correction term is grouped with the transport operator and is considered as a next order correction.

The so-called High-Field equilibrium can now be determined. Indeed, the energy brought into the system by the field is dissipated by the thermostat and allows to find a manifold of distribution functions (depending on two parameters, the density and the local temperature) which exactly balance the thermostatted field operator by the elastic collision operator.

As is frequent in this methodology, a general theory for the existence of HighField equilibria is hard to develop and we rather focus on two specific model collision operators: a one-dimensional BGK relaxation type operator (where the distribution function relaxes to a Maxwellian with same density and temperature) and a threedimensional Fokker-Planck operator (where again, the density and energy are preserved).

The resulting High-Field models consist of mass and energy balance equations which are first order nonlinear Partial Differential Equations. The mass and energy fluxes are just those of the above-mentioned High-Field equilibria and, in the special case considered, lead to explicit algebraic constitutive relations. The energy balance equation retains the work of the force field as an order $\varepsilon^{-1}$ term $(\varepsilon$ being the Knudsen number), as a reminiscence that the field was considered large enough to balance the collisions. Therefore, the solution of the model can be viewed more as a formal asymptotic limit of the original kinetic distribution function than as a regular limit. However, in realistic situations, $\varepsilon$ has a definite value, and it is not more complicated to deal with this term within the present model than within the classical EnergyTransport model.

Of course, one could try to continue the expansion and compute the next order terms, i.e. the $O(\varepsilon)$ diffusive corrections. This computation will be developed in a future work together with a proof that, for $O(\varepsilon)$ fields, the model gives back the standard Energy-Transport model. However, already at leading order the well-posedness of the models is not obvious. We give an answer to this question in the case of a given external electric field by showing that the systems are hyperbolic. Of course, when the electric field is coupled to the Poisson equation, more investigations are needed since another source of nonlinearity appears.

The paper is organized as follows. In section 2, we give a presentation of the thermostat concept and adapt it in the kinetic modeling setting. In section 3, we consider a one-dimensional thermostatted BGK equation, and introduce the HighField scaling for this equation. The associated High-Field fluid model is formally derived and the hyperbolicity of the model is proved. 
In section 4 , a more realistic $d$-dimensional model is considered. Here the considered collision operator is of Fokker-Planck type and two types of thermostats are examined, an isotropic thermostat and a thermostat acting only in the direction of the electric field $E$. The stationary solutions for both thermostats can be analytically computed and consist of a displaced Maxwellian (whose mean velocity is parallel to $E)$ in the case of the isotropic thermostat and an anisotropic Gaussian whose mean velocity is parallel to $E$ but whose thermal velocity is smaller in the direction parallel to $E$ in the case of the parallel thermostat. The stationary solution for the parallel thermostat has a remarkable energy equipartition property: all directions (including the one parallel to $E$ ) carry the same amount of energy, while those of the isotropic thermostat carry more energy in the $E$ direction. The corresponding fluid models are obtained and their hyperbolicity is proved. The properties of the two thermostats are discussed, and in view of this discussion the isotropic thermostat is preferred. The paper ends with a concluding section 5 .

\section{Thermostats}

In this section we present the thermostatted particle systems that present the microscopic picture of our models.

Consider first a system of $N$ identical particles with mass $m$, moving in one dimension under the influence of a (scalar) force field $E$. The equations are then, of course,

$$
\begin{aligned}
\frac{d}{d t} x_{j}(t) & =v_{j}(t), \\
m \frac{d}{d t} v_{j}(t) & =E, \quad j=1, \cdots, N .
\end{aligned}
$$

We are interested in very large systems of particles that, in addition to a force field as above, are subject to collisions, both among themselves and with the environment.

If the number of particles per unit length is very large (where the length scale is defined in terms of the gradient of $E$ ), it is relevant to consider a thermostat acting locally, i.e. on particles belonging to a small neighborhood of the position $x$. Therefore, we only deal with the velocities of the particles, having in mind that all these particles are localized in space around the point $x$. Similarly, we assume that the electric field is locally constant, which means that the potential energy is locally linear.

The total energy of this (local) system is

$$
\sum_{j=1}^{N}\left(\frac{1}{2} m v_{j}(t)^{2}-E x_{j}(t)\right)
$$

which, of course, is constant in a homogeneous situation. However, the kinetic energy is not; the particles will accelerate infinitely. In some cases it is interesting to modify the field so as to obtain a constant kinetic energy (see e.g. [42] and references therein). These are the so-called isokinetic thermostats. For a particle system moving in $\mathbb{R}^{d}$, the velocity component of the phase space is $\mathbb{R}^{d \times N}$, and the surface of constant kinetic energy is given by

$$
S_{W}=\left\{\left(v_{1}, \ldots, v_{N}\right): \sum_{j=1}^{N}\left|v_{j}\right|^{2}=N W\right\}
$$


and an iso-kinetic thermostat projects the force field $\mathbf{E}=(E, \ldots, E)$ onto the tangent space of $S_{W}$. A Gaussian iso-kinetic thermostat is obtained by choosing the orthogonal projection, which corresponds to Gauss' principle of minimal constraint force for nonholonomic constraints in mechanics. For the system (2.1), the Gaussian iso-kinetic thermostat takes the form (from now on, we set the particle mass equal to one)

$$
\dot{v}_{j}=E-\frac{E \cdot \sum_{k=1}^{N} v_{k}}{\sum_{k=1}^{N}|v|^{2}} v_{j}=E-\frac{E \cdot J}{2 W} v_{j},
$$

where $J=\sum_{k=1}^{N} v_{j}$, and $W=\frac{1}{2} \sum_{k=1}^{N}\left|v_{j}\right|^{2}$,

An alternative to the isotropic thermostat defined in (2.3) is to modify the field in the direction parallel to $E$ only. To this end we define

$$
W_{\|}=\frac{1}{2} \sum_{k=1}^{N} \frac{\left(E \cdot v_{k}\right)^{2}}{|E|^{2}},
$$

and then set

$$
\dot{v}_{j}=E-\frac{(E \cdot J)\left(E \cdot v_{j}\right)}{2 W_{\|}|E|^{2}} E .
$$

In Section 3 and 4, we consider kinetic equations where the particles are accelerated by thermostatted force fields, and in particular, we derive the corresponding fluid models. In particular, we will see, in Section 4, that the two different thermostats give a qualitative difference in the kinetic equations.

\section{A one-dimensional model}

3.1. Definitions and motivations. The starting point in this section is the following one-dimensional kinetic equation:

$$
\partial_{t} f+v \partial_{x} f+\partial_{v}(E f)=Q(f) .
$$

Here, $f=f(t, x, v)$ is the phase-space distribution function, $x, v \in \mathbb{R}$ are the onedimensional position and velocity, and $t \geq 0$ is the time. The force field $E=E(x, t)$ is supposed to be known and independent of $v$. However, the case where $E$ is dependent of $v$ is a straightforward extension and is left to the reader. We can also allow for self consistent force fields such as an electric field which would be a solution of Poisson's equation. Again, this extension is left to the reader.

To allow for explicit calculations, we take a one-dimensional BGK-operator with constant collision frequency,

$$
Q(f)(v)=\nu\left(n M_{T}(v)-f(v)\right) .
$$

Here $\nu$ is the collision frequency, and $M_{T}(v)$ is a centered Maxwellian with variance $T$, i.e.,

$$
M_{T}(v)=\frac{1}{\sqrt{2 \pi T}} \exp \left(-\frac{v^{2}}{2 T}\right) .
$$

The functions $n$ and $T$ are determined by

$$
\begin{aligned}
n(t, x) & =\int_{\mathbb{R}} f(t, x, v) d v \\
n(t, x) T(t, x) & =2 W(t, x)=\int_{\mathbb{R}} f(t, x, v) v^{2} d v .
\end{aligned}
$$


This implies in particular that

$$
\int_{\mathbb{R}} f d v=n \int_{\mathbb{R}} M_{T} d v \quad \text { and } \quad \int_{\mathbb{R}} f v^{2} d v=n \int_{\mathbb{R}} M_{T} v^{2} d v,
$$

or, in other words, the BGK operator (3.2) conserves mass and energy:

$$
\int_{\mathbb{R}} Q(f)(v) d v=0 \int_{\mathbb{R}} Q(f)(v)|v|^{2} d v=0 .
$$

Operator (3.2) is a toy model of realistic collision operators in semiconductor or plasma physics. In the semiconductor case, the actual collision operator is the sum of the phonon collision operator (in the elastic limit, i.e. when one neglects the phonon energy as compared with the typical electron energy) and of the electron-electron collision operator [3]. The resulting operator preserves mass and energy, exactly like (3.6), but not momentum (as the phonon collision operator does not preserve momentum). In plasmas, a similar consideration holds for the electrons against the neutrals and the ions. However, against the ions, the operator is more of a FokkerPlanck type form, and is best modeled by the Fokker-Planck operators of section 4 .

The left-hand side of equation (3.1) is the kinetic equation corresponding to the equation (2.1), and because the collision term in the right hand side does not dissipate energy, the total kinetic energy will be increasing. With a thermostatted force field (equation $(2.3)$ ), the term $\partial_{v} \cdot(E f)$ is replaced by $\partial_{v} \cdot(E f)-\mathcal{T}_{E}(f)$, where

$$
\mathcal{T}_{E}(f)=\partial_{v}\left(\frac{E j}{2 W} v f\right),
$$

where $W$ is as defined above, and the current $j$ is defined by

$$
j=\int_{\mathbb{R}} v f d v .
$$

3.2. Rescaling and the strong field approximation. We first review the standard diffusion scaling and the obstructions to making a High-Field Ansatz.

Investigating the large-scale behavior of particle systems described by eq. (3.1) first requires a change of time and length of diffusion type: $x^{\prime}=\epsilon x, t^{\prime}=\epsilon^{2} t, E=$ $\epsilon E^{\prime}$. The new space and time variables (those bearing the primes) are macroscopic variables, which vary of the order of unity when the old ones vary of order $1 / \epsilon$ or $1 / \epsilon^{2}$. In these new variables, eq. (3.1) is recast into (omitting the primes):

$$
\partial_{t} f^{\epsilon}+\frac{1}{\epsilon}\left(v \partial_{x} f^{\epsilon}+\partial_{v}\left(E f^{\epsilon}\right)\right)=\frac{1}{\epsilon^{2}} Q\left(f^{\epsilon}\right) .
$$

In the limit $\epsilon \rightarrow 0, f^{\epsilon}$ converges to an element of the null-space of $Q$, i.e. a Maxwellian of the form (3.3), whose density $n(x, t)$ and temperature $T(x, t)$ satisfy a system of coupled drift-diffusion equations, named as the Energy-Transport model (see [44], [30]). The determination of the model follows from the application of standard perturbation techniques named as the Hilbert or Chapman-Enskog expansions [13], [24], [33]. A diffusion system is obtained in the limit $\epsilon \rightarrow 0$, because the leading order Maxwellian has zero mean-velocity. Therefore, mean velocities are of order $\epsilon$ compared with the thermal velocity, which is typical of a diffusion process. 
For the diffusion scaling to be valid, the force field $E$ must be small of order $\epsilon$ (as apparent in the change of scale to $E^{\prime}$ ). In strongly non-equilibrium situations, this assumption is not valid and the field term becomes of the same order as the collision term (High-Field regime also denoted by drift-collision balance). In this regime, there is no change of scale on the field to be done. Also, because the drift due to the field becomes important, mean velocities become of order unity and a hydrodynamic scaling rather than a diffusion scaling is in order, setting $x^{\prime}=\epsilon x, t^{\prime}=\epsilon t$. Therefore, a High-Field scaling for (3.1) would read

$$
\partial_{t} f^{\epsilon}+v \partial_{x} f^{\epsilon}+\frac{1}{\epsilon} \partial_{v}\left(E f^{\epsilon}\right)=\frac{1}{\epsilon} Q\left(f^{\epsilon}\right) .
$$

In taking the limit $\epsilon \rightarrow 0$, the first task is to identify the solutions of the homogeneous leading order equation

$$
\tilde{Q}_{E}(f):=0, \quad Q_{E}(f)=Q(f)-\partial_{v}(E f),
$$

where the operator $\tilde{Q}_{E}(f)$ is the sum of the collision operator and the field operator and depends parametrically on the constant field $E$. By looking for solutions of (3.11), we are looking for distribution functions in which the collision operator is not zero but balances the field operator (so-called drift-collision balance). By analogy with the High-Field scaling of the Drift-Diffusion model [12], [45], [46], [47], one would expect that the solutions of (3.11) make an $E$-dependent manifold of dimension 2, parameterized by two quantities which might be viewed as High-Field generalizations of the chemical potential and temperature. The trouble is that no solution of (3.11) with finite energy can be found.

Indeed, integrating (3.11) with respect to velocity against $v$ and $v^{2}$ and using (3.2), we find

$$
0=-\nu j+E n, \quad E j=0 .
$$

Therefore, if $E \neq 0$ then successively $j=0$ and $n=0$. Then, since the distribution function is a positive quantity, we eventually find $f=0$. This is due to the fact that the energy increase in the system due to the field $E j$ is not balanced by any energy dissipation from the elastic collision operator. Another way to see this is to look at the homogeneous equation

$$
\partial_{t} f=\tilde{Q}_{E}(f),\left.\quad f\right|_{t=0}=f_{0},
$$

and to compute the evolution equation for the energy $W(t)$. We get the following evolution equations

$$
\partial_{t} n=0, \quad \partial_{t} j+\nu j=E n, \quad \partial_{t} W=E j .
$$

Therefore, the current $j$ converges exponentially fast to the constant value $j_{\infty}=E n / \nu$ as $t \rightarrow \infty$ and consequently, $W$ grows asymptotically linearly. Thus, the energy does not converge to a finite value in time, which is the signature of the fact that the energy increase due to the field is not balanced by the collisions.

Because of this difficulty, there is no High-Field Energy-Transport model like there are High-Field Drift-Diffusion models. The present paper represents, to our knowledge, the first attempt to overcome this difficulty. To do so, we introduce a thermostat and look for stationary solutions of the thermostatted field operator 
instead of the bare field operator. By using an iso-kinetic thermostat, we correct the energy increase of the field operator by a corresponding energy loss due to the thermostat, in such a way that the overall operator $\tilde{Q}_{E}$ preserves energy (and not only the bare collision operator $Q$ ).

We start by adding and subtracting the thermostat operator to the kinetic equation (3.1):

$$
\partial_{t} f+v \partial_{x} f+\mathcal{T}_{E}(f)=-E \partial_{v} f+\mathcal{T}_{E}(f)+Q(f),
$$

where $\mathcal{T}_{E}(f)$ is the thermostat operator (3.7). Then, we make a High-Field rescaling $x^{\prime}=\epsilon x, t^{\prime}=\epsilon t$ while keeping the field $E$ of order unity. The rescaled kinetic equation is written: $\epsilon \rightarrow 0$ :

$$
\epsilon\left(\partial_{t} f^{\epsilon}+v \partial_{x} f^{\epsilon}+\frac{1}{\epsilon} \mathcal{T}_{E}\left(f^{\epsilon}\right)\right)=-E \partial_{v} f^{\epsilon}+\mathcal{T}_{E}\left(f^{\epsilon}\right)+Q\left(f^{\epsilon}\right) .
$$

In investigating the High-Field limit $\epsilon \rightarrow 0$ of this equation, we shall make the assumption that the thermostat operator at the right-hand side is of order 1 , the thermostat operator at while the thermostat operator at the left-hand side is of order $\epsilon$.

Of course, from a strict mathematical standpoint, it is inconsistent to have the same thermostat operator appearing at different orders. In an attempt to make this approach more consistent, we split the parameter $\epsilon$ into two parameters $\epsilon$ and $\eta$ and transform eq. (3.13) into the following:

$$
\epsilon\left(\partial_{t} f^{\epsilon}+v \partial_{x} f^{\epsilon}+\frac{1}{\eta} \mathcal{T}_{E}\left(f^{\epsilon}\right)\right)=-E \partial_{v} f^{\epsilon}+\mathcal{T}_{E}\left(f^{\epsilon}\right)+Q\left(f^{\epsilon}\right) .
$$

Of course, (3.14) is only equivalent to (3.13) if $\eta=\epsilon$. On the other hand, it is legitimate, from a mathematical viewpoint, to investigate the limit $\epsilon \rightarrow 0$ in (3.14) while keeping $\eta$ fixed. Eventually, once the macroscopic system has been derived, we must bear in mind that $\eta=\epsilon$.

At leading order, when $\varepsilon \rightarrow 0$ with $\eta$ kept fixed, $f$ solves the homogeneous stationary equation

$$
Q_{E}(f):=-E \partial_{v} f+\mathcal{T}_{E}(f)+Q(f)=0 .
$$

This stationary equation involves a composite operator made of the field operator corrected by its thermostat and the collision operator.

Obviously, the thermostat operator at the left-hand side of (3.14) contributes to the macroscopic energy balance law by a term of order $1 / \eta$ representing the work of the electric force. Therefore, if $\eta=\epsilon$ in the resulting macroscopic equations, the correct magnitude of this term is recovered. The only change that this approach provides compared to the conventional one is that, in the limit $\epsilon \rightarrow 0$, the leading order distribution function is a solution of (3.15) rather than a mere Maxwellian. Because (3.15) carries information about the field, we expect this solution to be closer to the actual $f^{\epsilon}$ than the Maxwellian. Of course, while this assertion is certainly true from a mathematical viewpoint if $\eta$ is kept fixed as $\epsilon \rightarrow 0$, it is far less obvious (and certainly hard to formalize mathematically) if $\eta$ has the correct value $\eta=\epsilon$. Nonetheless, we have physical reasons to believe that this approach will provide an improved model as compared with the standard approach. Additionally, when $E$ is weak, the solution of (3.15) is actually close to that of $Q(f)=0$, i.e. is close to a Maxwellian. Thus, we expect that the solution of (3.15) is some kind of perturbation of the Maxwellian, 
which takes into account the deformation of the distribution function in the direction of the field.

We shall see that (3.15) is actually solvable and its solution space is a two-dimensional manifold.

Since the solution of (3.15) is parametrized by the field, the associated mass and energy fluxes are also field dependent. Therefore, this approach provides a justification (and a practical computational methodology) to the field-dependent mass and energy fluxes that are used in practice and which are, so far, based on phenomenological considerations. In the Drift-Diffusion case, this approach (without the need of thermostats) provides field-dependent expressions of the carrier mobilities which are closer to the experimentally measured ones than those predicted by the Low-Field scaling (3.9) (see [4], [22]). Similarly, here, we also expect that these expression can lead to a better fit of the experimental values than the field-independent expressions obtained by the Low-Field scaling (3.9).

3.3. The leading order distribution in the High-Field approximation.

We now investigate the limit $\epsilon \rightarrow 0$ of (3.14). We suppose that $f^{\epsilon} \rightarrow f$ as smoothly as needed. Then at leading order, $f$ solves (3.15). In this section, we investigate the solvability of (3.15). Inserting the expression (3.2) of $Q,(3.15)$ is written:

$$
-E \partial_{v} f+\mathcal{T}_{E}(f)+\nu n M_{T}-\nu f=0,
$$

or, using the expression (3.7) for $\mathcal{T}_{E}(f)$ and setting $j=n u$ :

$$
-E \partial_{v}\left(\left(1-\frac{n u v}{2 W}\right) f\right)+\nu n M_{T}-\nu f=0 .
$$

By homogeneity we may assume that $n=\int_{\mathbb{R}} f(v) d v=1$, and transforming to nondimensional variables,

$$
\begin{aligned}
\tilde{v} & =v / \sqrt{T}, \\
\tilde{u} & =u / \sqrt{T}, \\
\tilde{f}(\tilde{v}) & =T^{1 / 2} f(\sqrt{T} \tilde{v}), \\
\tilde{E} & =E / \nu \sqrt{T}, \\
\tilde{W} & =W / T,
\end{aligned}
$$

leads to the equation

$$
-\tilde{E} \partial_{\tilde{v}}\left(\left(\frac{1-\tilde{u} \tilde{v}}{2 \tilde{W}}\right) \tilde{f}\right)+M_{1}-\tilde{f}=0 .
$$

It is almost obvious from the construction that $2 \tilde{W}=1$, and it can be checked by multiplying (3.19) by $|\tilde{v}|^{2}$ and integrating.

In this particular case, where $Q$ is a BGK-type kernel, equation (3.19) can be solved almost explicitly. After dividing by $\tilde{E}(1-\tilde{u} \tilde{v})$,

$$
\partial_{\tilde{v}} \tilde{f}-\frac{\tilde{E} \tilde{u}-1}{\tilde{E}(1-\tilde{u} \tilde{v})} \tilde{f}=\frac{1}{\tilde{E}(1-\tilde{u} \tilde{v})} M_{1} .
$$

An integrating factor for this ordinary differential equation is

$$
\frac{1-\tilde{E} \tilde{u}}{\tilde{E} \tilde{u}} \log \left|\tilde{v}-\frac{1}{\tilde{u}}\right|=\log \left(\left|\tilde{v}-\frac{1}{\tilde{u}}\right|^{\frac{1}{\tilde{E} \tilde{u}}-1}\right) \text {. }
$$


The mean velocity $\tilde{u}$ for the stationary problem depends on $\tilde{E}$, and to find this relation multiply by $\tilde{v}$ and integrate, to get

$$
\tilde{E}\left(1-\tilde{u}^{2}\right)-\tilde{u}=0,
$$

or

$$
\tilde{u}=\frac{-1 \pm \sqrt{1+4 \tilde{E}^{2}}}{2 \tilde{E}}=\frac{\sqrt{1+4 \tilde{E}^{2}}-1}{2 \tilde{E}} .
$$

(Only this solution satisfies the energy constraint.) We also see that $\tilde{E} \tilde{u}=1 \Leftrightarrow \tilde{E}=\sqrt{2}$, and that

$$
\tilde{E}<\sqrt{2} \Rightarrow \frac{1}{\tilde{E} \tilde{u}}-1>0 \quad \text { and } \quad \tilde{E}>\sqrt{2} \Rightarrow \frac{1}{\tilde{E} \tilde{u}}-1<0 .
$$

Equation (3.20) can be solved using the integrating factor, and the result, $\tilde{f}_{E}(v)$, which depends on the parameter $\tilde{E}$, can be written

$$
\tilde{f}_{\tilde{E}}(\tilde{v})=\frac{1}{\tilde{E} \tilde{u}}\left|\tilde{v}-\frac{1}{\tilde{u}}\right|^{\frac{1}{\tilde{E} \tilde{u}}}-1 \begin{cases}\int_{-\infty}^{\tilde{v}} M(w)\left|w-\frac{1}{\tilde{u}}\right|^{-\frac{1}{\tilde{E} \tilde{u}}} d w & \tilde{v}<1 / \tilde{u} \\ \int_{\tilde{v}}^{\infty} M(w)\left|w-\frac{1}{\tilde{u}}\right|^{-\frac{1}{\tilde{E}}} d w & \tilde{v}>1 / \tilde{u} .\end{cases}
$$

Finally, the normalized (i.e. with total integral unity) leading order distribution has the following expression:

$$
F_{E, T}(v)=\frac{1}{\sqrt{T}} \tilde{f}_{E / \nu \sqrt{T}}(v / \sqrt{T}) .
$$

3.4. The fluid equations. From the previous section, the limit $f$ of $f^{\epsilon}$ when $\epsilon \rightarrow 0$ is of the form

$$
f=n F_{E, T}(v),
$$

where $n>0$ and $T>0$ are free parameters, such that

$$
\int_{\mathbb{R}} f\left(\begin{array}{c}
1 \\
|v|^{2}
\end{array}\right) d v=\left(\begin{array}{c}
n \\
W=\frac{1}{2} n T
\end{array}\right),
$$

and where again, $n$ is the particle density and $W$ the energy density.

Eq. (3.17) is a homogeneous equation, and does not imply anything about the dependence of $f$ upon $(x, t)$. Therefore, the parameters $n$ and $T$ are functions of $(x, t)$. Again, the force field $E(x, t)$ is supposed known and a function of $(x, t)$ in general. To determine $n$ and $T$, we return to (3.14) and use the fact that the operator $Q_{E}$ defined by (3.15) is mass- and energy-conservative (the latter being the consequence of the addition of the thermostat), i.e. $Q_{E}$ satisfies

$$
\int_{\mathbb{R}} Q_{E}(f)\left(\begin{array}{c}
1 \\
|v|^{2}
\end{array}\right) d v=0 .
$$


Therefore, integrating (3.14) with respect to $v$ after multiplication by 1 and $|v|^{2}$ we get

$$
\begin{aligned}
& \partial_{t} n^{\epsilon}(x, t)+\partial_{x} j^{\epsilon}(x, t)=0 \\
& \partial_{t} W^{\epsilon}(x, t)+\partial_{x} j_{W}^{\epsilon}(x, t)+\frac{1}{2 \eta} \int_{\mathbb{R}} v^{2} \partial_{v}\left(\frac{j^{\epsilon} E}{2 W^{\epsilon}} v f^{\epsilon}\right) d v=0
\end{aligned}
$$

where $n^{\epsilon}, W^{\epsilon}, j^{\epsilon}$ are respectively the density, energy and particle current associated with $f^{\epsilon}$ according to (3.4), (3.5), (3.8), and

$$
j_{W}^{\epsilon}=\frac{1}{2} \int_{\mathbb{R}} f^{\epsilon}(x, v, t) v^{3} d v
$$

is the energy current. We note that the conservation equations (3.30), (3.31) are satisfied $\epsilon$ being zero or not. They express the general laws of local conservation of mass and energy. We first note that the integral term in (3.31) (which is due to the thermostat) can be computed directly by integration by parts:

$$
\frac{1}{2} \int_{\mathbb{R}} v^{2} \partial_{v}\left(\frac{j^{\epsilon} E}{2 W^{\epsilon}} v f^{\epsilon}\right) d v=-j^{\epsilon} E .
$$

Therefore, the energy conservation equation is also written:

$$
\partial_{t} W^{\epsilon}(x, t)+\partial_{x} j_{W}^{\epsilon}(x, t)=\frac{1}{\eta} j^{\epsilon} E .
$$

The term at the right hand-side is nothing but the work of the electric force (responsible for Joule heating). In the original physical model $\eta=\epsilon$. Therefore, the Joule heating term is of order $1 / \epsilon$ because the field is of this order.

Now, in the limit $\epsilon \rightarrow 0$, we have $f^{\epsilon} \rightarrow f$ (formally) where $f$ is given by (3.27). Therefore, in taking the limit $\epsilon \rightarrow 0$ in (3.30), (3.34), we can express the fluxes $j$ and $j_{W}$ in terms of $n, T$ and $E$ by using (3.27). We now perform this computation.

Note that in physical variables, equation (3.23) becomes

$$
\frac{u}{\sqrt{T}}=\frac{\sqrt{1+4 E^{2} / \nu^{2} T}-1}{2 E / \sqrt{\nu^{2} T}}
$$

which means that no equation is needed for the first moment of $f$;

$$
j=n u=n \sqrt{T} \frac{\sqrt{\nu^{2} T+4 E^{2}}-\sqrt{\nu^{2} T}}{2 E} .
$$

The energy flux is given by

$$
j_{W}=\frac{1}{2} \int_{\mathbb{R}} f(x, v, t) v^{3} d v=\frac{1}{2} n T^{3 / 2} \int_{\mathbb{R}} \tilde{f} \tilde{v}^{3} d \tilde{v}=n T^{3 / 2} \tilde{j}_{W},
$$

where $\tilde{j}_{W}$ can be obtained by multiplying equation (3.19) by $\tilde{v}^{3}$ and integrating:

$$
\int_{\mathbb{R}} 3 \tilde{E} \tilde{v}^{2}(1-\tilde{u} \tilde{v}) \tilde{f} d \tilde{v}+\int_{\mathbb{R}} M_{1}(\tilde{v}) \tilde{v}^{3} d \tilde{v}-\int_{\mathbb{R}} \tilde{v}^{3} \tilde{f} d \tilde{v}=0,
$$

i.e.

$$
3 \tilde{E}\left(\frac{1}{2}-\tilde{u} \tilde{j}_{W}\right)-\tilde{j}_{W}=0,
$$


or

$$
\tilde{j}_{W}=\frac{3 \tilde{E}}{2(1+3 \tilde{E} \tilde{u})}
$$

Using equation $(3.23)$ gives $1+3 \tilde{E} \tilde{u}=\frac{1}{2}\left(3 \sqrt{1+4 \tilde{E}^{2}}-1\right)$, and then

$$
\tilde{j}_{W}=\frac{3}{4} \frac{\tilde{E}\left(3 \sqrt{1+4 \tilde{E}^{2}}+1\right)}{2+9 \tilde{E}^{2}},
$$

and in physical variables

$$
j_{W}=\frac{3}{4} n T^{3 / 2} \frac{E\left(3 \sqrt{\nu^{2} T+4 E^{2}}+\sqrt{\nu^{2} T}\right)}{2 \nu^{2} T+9 E^{2}} .
$$

In summary, writing $j_{W}=\frac{1}{2} n T u_{W}$, where $u_{W}$ is the convection velocity for the energy $W=\frac{1}{2} n T$, the full, limiting system becomes

$$
\left\{\begin{array}{l}
\partial_{t} n+\partial_{x}(n u)=0 \\
\partial_{t} W+\partial_{x}\left(W u_{W}\right)-\frac{1}{\eta} E n u=0 \\
u=\sqrt{T} \frac{\sqrt{\nu^{2} T+4 E^{2}}-\sqrt{\nu^{2} T}}{2 E} \\
u_{W}=\frac{3}{2} \sqrt{T} \frac{E\left(3 \sqrt{\nu^{2} T+4 E^{2}}+\sqrt{\nu^{2} T}\right)}{2 \nu^{2} T+9 E^{2}} \\
W=\frac{1}{2} n T
\end{array} .\right.
$$

Back to the original physical problem, we must set $\eta=\epsilon$.

3.5. Hyperbolicity of the limiting system. We first write system (3.40) in an abstract way.

Let $U=\left(\begin{array}{c}n \\ W\end{array}\right), f(U)=\left(\begin{array}{c}n u \\ W u_{W}\end{array}\right)$, and $g(U)=\left(\begin{array}{c}0 \\ \frac{1}{\eta} E n u\end{array}\right)$.

Then (3.40) becomes

$$
\partial_{t} U+\partial_{x} f(U)=g(U)
$$

and proving the hyperbolicity of the system amounts to showing that $f^{\prime}(U)$ has two different real eigenvalues. Since $W=\frac{1}{2} n T$, we may write, for a function of $T$ only, $\partial_{n}=\left(-\frac{2 W}{n^{2}}\right) \partial_{T}$, and $\partial_{W}=\left(\frac{2}{n}\right) \partial_{T}$, and hence

$$
\begin{aligned}
f^{\prime}(U) & =\left(\begin{array}{cc}
u+n\left(-\frac{2 W}{n^{2}}\right) \partial_{T} u & n\left(\frac{2}{n}\right) \partial_{T} u \\
W\left(-2 \frac{W}{n^{2}}\right) \partial_{T} u_{W} & u_{W}+W \frac{2}{n} \partial_{T} u_{W}
\end{array}\right) \\
& =\left(\begin{array}{cc}
u-T \partial_{T} u & 2 \partial_{T} u \\
-\frac{T}{2} \partial_{T} u_{W} & u_{W}+T \partial_{T} u_{W}
\end{array}\right)
\end{aligned}
$$


Therefore, the characteristic polynomial of $f^{\prime}(U)$ has the following expression:

$$
\begin{aligned}
P(\lambda):= & \operatorname{det}\left(f^{\prime}(U)-\lambda I\right) \\
= & \left(u-T \partial_{T} u-\lambda\right)\left(u_{W}+T \partial_{T} u_{W}-\lambda\right)+T^{2} \partial_{T} u \partial_{T} u_{W} \\
= & \lambda^{2}-\lambda\left(u-T \partial_{T} u+u_{W}+T \partial_{T} u_{W}\right) \\
& +\left(u-T \partial_{T} u\right)\left(u_{W}+T \partial_{T} u_{W}\right)+T^{2} \partial_{T} u \partial_{T} u_{W} .
\end{aligned}
$$

The hyperbolicity of the system (3.40) can then be established by showing that the discriminant $\Delta$ to $P(\lambda)$ is positive.

We have by elementary manipulations:

$$
\begin{aligned}
\Delta= & \left(u-T \partial_{T} u+u_{W}+T \partial_{T} u_{W}\right)^{2} \\
& -4\left[\left(u-T \partial_{T} u\right)\left(u_{W}+T \partial_{T} u_{W}\right)+T^{2} \partial_{T} u \partial_{T} u_{W}\right] \\
= & \left(u-T \partial_{T} u-u_{W}-T \partial_{T} u_{W}\right)^{2}-4 T^{2} \partial_{T} u \partial_{T} u_{W} \\
= & \left(u-u_{W}\right)^{2}+\left(T \partial_{T}\left(u+u_{W}\right)\right)^{2}-2\left(u-u_{W}\right)\left(T \partial_{T}\left(u+u_{W}\right)\right) \\
& -4 T^{2} \partial_{T} u \partial_{T} u_{W} \\
= & \left(u-u_{W}\right)^{2}+\left(T \partial_{T}\left(u-u_{W}\right)\right)^{2}-2\left(u-u_{W}\right)\left(T \partial_{T}\left(u+u_{W}\right)\right) .
\end{aligned}
$$

Now, in this last equality, the first two terms are obviously positive. We are going to show that the last term is positive by showing that $u$ and $u_{W}$ are increasing functions of $T$ on the one hand and that $u<u_{W}$ on the other hand.

For this purpose, we introduce the short-hand notations $z=\nu \sqrt{T} /(2 E)$. We shall assume that $E>0$; the case $E<0$ can be easily deduced by a change of variables. Then:

$$
u=\frac{2 E}{\nu} z\left(\sqrt{z^{2}+1}-z\right), \quad u_{W}=\frac{6 E}{\nu} \frac{z\left(3 \sqrt{z^{2}+1}+z\right)}{8 z^{2}+9} .
$$

Now, it is a matter of elementary algebra to show that

$$
\begin{aligned}
u^{\prime}(z) & =\frac{2 E}{\nu} \frac{\left(\sqrt{z^{2}+1}-z\right)^{2}}{\sqrt{z^{2}+1}}>0, \\
u_{W}^{\prime}(z) & =\frac{6 E}{\nu} \frac{30 z^{2}+27+18 z \sqrt{z^{2}+1}}{\left(8 z^{2}+9\right)^{2} \sqrt{z^{2}+1}}>0,
\end{aligned}
$$

showing that $u$ and $u_{W}$ are increasing functions of $T$. Now, again, elementary algebra gives:

$$
\frac{u_{W}}{u}=1+\frac{4 z^{2}+12 z \sqrt{z^{2}+1}}{\left(8 z^{2}+9\right)}>1,
$$

showing that $u_{W}>u$. Therefore, $\Delta>0$ and the system is strictly hyperbolic.

The strict hyperbolicity of the system guarantees its well-posedness, at least when the electric field $E$ is given. If $E$ is coupled to $n$ through Poisson's equation, the wellposedness of the system is an open question. The complexity of the dependence of $u$ and $u_{W}$ upon $T$ makes it difficult to explore further properties of the system, such as showing that the two fields are genuinely nonlinear, but we conjecture that this is actually the case. 
3.6. Remarkable limit cases and monotonicity with respect to $E$.

We investigate the two remarkable limit cases $z \rightarrow \infty$ (Low-Field case) and $z \rightarrow 0$ (High-Field case).

(i) Low-Field case $z \rightarrow \infty$ : then

$$
u \sim \frac{E}{\nu}, \quad u_{W} \sim \frac{3 E}{\nu} .
$$

We recover mobility laws, with a Low-Field mobility equal to $\nu^{-1}$ as it should be and the mobility of the energy is three times the mobility for the density. We notice here that the energy velocity is larger than the particle velocity, which confirms our general result just proven above.

(ii) High-Field case $z \rightarrow 0$ : then

$$
u \sim \sqrt{T}, \quad u_{W} \sim \sqrt{T} .
$$

Here, the particle and energy velocities are equal and both are close to $\sqrt{T}$. We emphasize the fact that $\sqrt{T}$ should not be viewed as a thermal velocity because the energy satisfies $W=n T / 2$ which shows that $\sqrt{T}$ is the magnitude of the total velocity rather than magnitude of the thermal velocity. Indeed, in the High-Field case, the particle distribution function concentrates onto a delta function at velocity " $\sqrt{T}$ ", which explains why both the particle and energy velocities are equal to. If the next order correction term is retained, for large but not infinite field values, we find

$$
u \sim \sqrt{T}-\frac{\nu T}{2 E}, \quad u_{W} \sim \sqrt{T}+\frac{\nu T}{6 E} .
$$

Again, we see that as $E^{-1}$ increases, the energy velocity is larger than $\sqrt{T}$ while the particle velocity is smaller, and in particular we again find that $u<u_{W}$.

Another interesting consideration is to look for the monotonicity with respect to $E$. For this purpose, we introduce $y=\frac{2 E}{\nu \sqrt{T}}=\frac{1}{z}$ and write

$$
\begin{gathered}
u=\sqrt{T} \frac{1}{y}\left(\sqrt{1+y^{2}}-1\right)=\sqrt{T} \frac{1}{\sqrt{\frac{1}{y^{2}}+1}+\frac{1}{y}}, \\
u_{W}=3 \sqrt{T} y \frac{3 \sqrt{1+y^{2}}+1}{4+9 y^{2}}=3 \sqrt{T}\left(3 \sqrt{1+y^{2}}+1\right) \frac{1}{\frac{4}{y^{2}}+9} .
\end{gathered}
$$

Both functions at the right-hand sides of (3.42) and (3.43) are increasing w.r.t.y, showing that both $u$ and $u_{W}$ are monotonically increasing functions of the electric field. The limit cases $E$ small and $E$ large have already been given above.

\section{A $d$-dimensional model: the Fokker-Planck equation}

4.1. Setting of the model. In this section we consider equation (3.14) in three dimensions and with the Fokker-Planck collision operator:

$$
Q(f)=Q_{\mathrm{fp}}(f)=\nu \nabla_{v} \cdot\left(v f+T \nabla_{v} f\right),
$$


where the temperature $T$ is related to $f$ through

$$
d n T=2 W=\int_{\mathbb{R}^{d}} f|v|^{2} d v, \quad n=\int_{\mathbb{R}^{d}} f d v .
$$

The collision frequency $\nu$ is supposed constant. In this form, it conserves mass and energy, i.e.

$$
\int_{\mathbb{R}^{d}} Q_{\mathrm{fp}}(f)(v) d v=0 \quad \int_{\mathbb{R}^{d}} Q_{\mathrm{fp}}(f)(v)|v|^{2} d v=0,
$$

but not momentum, i.e. $\int Q_{\mathrm{fp}}(f)(v) v d v \neq 0$.

There is obviously no unique way in which a thermostat can be introduced, and here we consider first the natural, isotropic thermostat, corresponding to (3.7),

$$
\mathcal{T}_{E, \text { iso }}=\nabla_{v} \cdot\left(\frac{E \cdot j}{2 W} v f\right),
$$

and a thermostat that acts only parallel to the (constant) field $E$,

$$
\mathcal{T}_{E, \|}=\nabla_{v} \cdot\left(\frac{E \cdot j}{2 W_{\|}} \frac{(E \cdot v) E}{|E|^{2}} f\right)
$$

where

$$
W=\frac{1}{2} \int_{\mathbb{R}^{d}}|v|^{2} f d v \quad \text { and } \quad W_{\|}=\frac{1}{2} \int_{\mathbb{R}^{d}} \frac{|E \cdot v|^{2}}{|E|^{2}} f d v,
$$

and where

$$
j=n u=\int_{\mathbb{R}^{d}} v f d v .
$$

It is easy to check that both $\mathcal{T}_{E \text {,iso }}$ and $\mathcal{T}_{E, \|}$ accomplish energy conservation:

$$
\int_{\mathbb{R}^{d}}\left(\nabla_{v} \cdot(E f)-\mathcal{T}_{E, \text { iso }}(f)\right)|v|^{2} d v=\int_{\mathbb{R}^{d}}\left(\nabla_{v} \cdot(E f)-\mathcal{T}_{E, \|}(f)\right)|v|^{2} d v=0 .
$$

In this section, we are interested in finding formally the asymptotics of the kinetic equation

$$
\epsilon\left(\partial_{t} f^{\epsilon}+v \cdot \nabla_{x} f^{\epsilon}+\frac{1}{\epsilon} \mathcal{T}_{E}\left(f^{\epsilon}\right)\right)=-E \cdot \nabla_{v} f^{\epsilon}+\mathcal{T}_{E}\left(f^{\epsilon}\right)+Q_{\mathrm{fp}}\left(f^{\epsilon}\right)
$$

with $f^{\epsilon}=f^{\epsilon}(x, v, t), x \in \mathbb{R}^{d}, v \in \mathbb{R}^{d}$ and $t>0$, where $\mathcal{T}_{E}(f)$ is one of $\mathcal{T}_{E \text {,iso }}$ and $\mathcal{T}_{E, \|}$ and considering that the thermostat operator at the left-hand side is of order $\epsilon$ while that at the right-hand side is of order 1.

4.2. The leading order distribution in the High-Field approximation: general considerations. To derive fluid equations we first need to solve the zeroth order equations

$$
\nabla_{v} \cdot(E f)-\mathcal{T}_{E}(f)=\nu \nabla_{v} \cdot\left(v f+T \nabla_{v} f\right),
$$


where $\mathcal{T}_{E}(f)$ is one of $\mathcal{T}_{E \text {,iso }}$ and $\mathcal{T}_{E, \|}$. In both cases, one can immediately integrate once to obtain

$$
\begin{aligned}
E f-\frac{E \cdot j}{2 W} v f & =\nu v f+\nu T \nabla_{v} f \quad \text { and } \\
E f-\frac{E \cdot j}{2 W_{\|}} \frac{(E \cdot v) E}{|E|^{2}} f & =\nu v f+\nu T \nabla_{v} f
\end{aligned}
$$

respectively. The constant of integration must be zero because $f$ is supposed to be in $L^{1}$. These equations are homogeneous in $f$, and so there is no loss of generality at this point in assuming that $n=\int_{\mathbb{R}^{d}} f d v=1$. It is convenient to rewrite the equations in non-dimensional variables, using

$$
\begin{aligned}
& \tilde{v}=v / \sqrt{T} \quad \tilde{u}=u / \sqrt{T} \quad \tilde{j}=j / \sqrt{T} \\
& \tilde{f}(\tilde{v})=T^{d / 2} f(\sqrt{T} \tilde{v}) \quad \tilde{E}=E / \nu \sqrt{T} \\
& \tilde{W}=W / T \quad \tilde{W}_{\|}=W_{\|} / T
\end{aligned}
$$

and because $n=1, \tilde{j}=\tilde{u}$. In these variables, the equations (4.5) become

$$
\begin{aligned}
& \tilde{E} \tilde{f}-\frac{\tilde{E} \cdot \tilde{u}}{2 \tilde{W}} \tilde{v} \tilde{f}=\tilde{v} \tilde{f}+\nabla_{\tilde{v}} \tilde{f} \quad \text { and } \\
& \tilde{E} \tilde{f}-\frac{\tilde{E} \cdot \tilde{u}}{2 \tilde{W}_{\|}} \frac{(\tilde{E} \cdot \tilde{v}) \tilde{E}}{|\tilde{E}|^{2}} \tilde{f}=\tilde{v} \tilde{f}+\nabla_{\tilde{v}} \tilde{f} .
\end{aligned}
$$

Some conclusions may be drawn directly from these equations.

First of all, by integrating over $\mathbb{R}^{d}$ one may see that for both equations, the mean velocity $\tilde{u}$ is parallel to the field $\tilde{E}$, and of course the same holds in the dimensional variables. This is an effect of the strong field approximation, and will result in fluid equations that are essentially one-dimensional in $x$. The components of $x$ that are orthogonal to $E$ are only parameters, and we will find equations that are very similar to the genuinely one-dimensional case that was considered in Section 3.

Next, in both cases the left-hand side vanishes if the equations are multiplied by $\tilde{v}$ and integrated. This implies that

$$
0=\int_{\mathbb{R}^{d}}|\tilde{v}|^{2} \tilde{f} d \tilde{v}+\int_{\mathbb{R}^{d}} \tilde{v} \cdot \nabla_{\tilde{v}} \tilde{f} d \tilde{v}=2 \tilde{W}-d \int_{\mathbb{R}^{d}} \tilde{f} d \tilde{v}=2 \tilde{W}-d,
$$

just as one should expect from the scaling. Below, $2 \tilde{W}$ will hence be replaced by $d$. Next we multiply by $(\tilde{E} \cdot \tilde{v}) \tilde{E}$, and integrate. This gives for equation $(4.7 \mathrm{~b})$ that

$$
0=\int_{\mathbb{R}^{d}}(\tilde{E} \cdot \tilde{v})^{2} \tilde{f} d \tilde{v}+\int_{\mathbb{R}^{d}}(\tilde{E} \cdot \tilde{v}) \tilde{E} \cdot \nabla_{\tilde{v}} \tilde{f} d \tilde{v}=2|\tilde{E}|^{2} \tilde{W}_{\|}-|\tilde{E}|^{2} \int_{\mathbb{R}^{d}} \tilde{f} d \tilde{v},
$$

which implies that $2 \tilde{W}_{\|}=1$. This can be interpreted as a result on the equipartition of energy for a solution to (4.7b). For symmetry reasons, we must have $\int_{\mathbb{R}^{d}} \tilde{v}_{k}^{2} \tilde{f} d \tilde{v}=1$ for any $v_{k}$ orthogonal to $E$, and hence, for any vector $A \in \mathbb{R}^{d}$,

$$
\int_{\mathbb{R}^{d}}(A \cdot \tilde{v})^{2} \tilde{f} d \tilde{v}=|A|^{2} .
$$

On the other hand, multiplying $(4.7 \mathrm{a})$ with $(\tilde{E} \cdot \tilde{v}) \tilde{E}$, and integrating gives

$$
|\tilde{E}|^{2} \tilde{E} \cdot \tilde{u}-\frac{\tilde{E} \cdot \tilde{u}}{d} 2|\tilde{E}|^{2} \tilde{W}_{\|}=2|\tilde{E}|^{2} \tilde{W}_{\|}-|\tilde{E}|^{2},
$$


because the right-hand side is the same for the two equations. Then, for the isotropic thermostat,

$$
2 \tilde{W}_{\|}=\frac{\tilde{E} \cdot \tilde{u}+1}{\frac{\tilde{E} \cdot \tilde{u}}{d}+1}=\frac{d \tilde{E} \cdot \tilde{u}+d}{\tilde{E} \cdot \tilde{u}+d}>1,
$$

and for a strong field $E$ almost all kinetic energy lies in velocity components parallel to $\tilde{E}$.

4.3. The leading order distribution for an isotropic thermostat. First we rewrite $(4.7 \mathrm{a})$ as

$$
\nabla_{\tilde{v}} \tilde{f}=\tilde{E} f-\left(1+\frac{\tilde{E} \cdot \tilde{u}}{d}\right) \tilde{v} \tilde{f} .
$$

Then, multiplication with $\tilde{E}$ and integration yields

$$
0=|\tilde{E}|^{2}-\left(1+\frac{\tilde{E} \cdot \tilde{u}}{d}\right) \tilde{E} \cdot \tilde{u},
$$

and with

$$
\beta=1+\frac{\tilde{E} \cdot \tilde{u}}{d}
$$

this is the same as

$$
\beta^{2}-\beta-\frac{|\tilde{E}|^{2}}{d}=0
$$

or

$$
\beta=\frac{1+\sqrt{1+4|\tilde{E}|^{2} / d}}{2} .
$$

From (4.8) we now find

$$
\log \tilde{f}=C+\tilde{E} \tilde{v}-\frac{\beta}{2}|\tilde{v}|^{2}=C-\frac{\beta}{2}\left|\tilde{v}-\frac{\tilde{E}}{\beta}\right|^{2},
$$

i.e. the leading order distribution function

$$
\tilde{f}(\tilde{v})=\left(\frac{\beta}{2 \pi}\right)^{d / 2} \exp \left(-\frac{\beta}{2}\left|\tilde{v}-\frac{\tilde{E}}{\beta}\right|^{2}\right)
$$

is a Maxwellian with temperature $1 / \beta$, and displaced along $\tilde{E}$. Equation (4.12) shows that $1 / \beta \rightarrow 0$ when $\tilde{E} \rightarrow \infty$; for a very strong field, the Maxwellian is concentrated very near the point $v=d \tilde{E} /|\tilde{E}|$.

In the dimensional variables, the normalized leading order distribution is a Maxwellian which has the following expression:

$$
F_{E, T}^{\text {iso }}=\left(\frac{\beta}{2 \pi}\right)^{d / 2} \exp \left(-\frac{\beta}{2 T}\left|v-\frac{E}{\nu \beta}\right|^{2}\right),
$$

where

$$
\beta=\frac{\sqrt{d \nu^{2} T}+\sqrt{d \nu^{2} T+4|E|^{2}}}{2 \sqrt{d \nu^{2} T}} .
$$


4.4. The fluid equations for the isotropic thermostat. The first order fluid equations are obtained by a procedure similar to that exposited in section 3 for the one-dimensional case. The procedure will not be repeated here and only the result is given. The fluid equations are written:

$$
\begin{aligned}
& \partial_{t} n(x, t)+\nabla_{x} \cdot j(x, t)=0, \\
& \partial_{t} W(x, t)+\nabla_{x} \cdot j_{W}(x, t)=\frac{1}{\eta} E \cdot j d v,
\end{aligned}
$$

where we recall that the energy flux $j_{W}$ is given by

$$
j_{W}=\frac{1}{2} \int_{\mathbb{R}^{d}} f(x, v, t)|v|^{2} v d v .
$$

The mass and energy fluxes $j$ and $j_{W}$ are computed by inserting expression (4.14) for the leading order distribution $n F_{E, T}^{\text {iso }}$ into the integral definitions (4.3) and (4.16).

First, the mass flux is given by

$$
j=n u=n u_{\|} \frac{E}{|E|},
$$

and $u_{\|}$can be obtained from $\tilde{u}_{\|}=d(\beta-1) /|\tilde{E}|$ (see equation (4.10)), or directly from equation (4.14)

$$
\begin{aligned}
u=\frac{E}{\nu \beta} & =\frac{2 \sqrt{d \nu^{2} T}}{\sqrt{d \nu^{2} T}+\sqrt{d \nu^{2} T+4|E|^{2}}} \frac{E}{\nu} \\
& =\sqrt{d T} \frac{\sqrt{d \nu^{2} T+4|E|^{2}}-\sqrt{d \nu^{2} T}}{2|E|^{2}} E .
\end{aligned}
$$

Hence the component of $u$ parallel to $E$ is essentially the same as in the onedimensional case considered in Section 3.

The expression of the energy flux $j_{W}$ can be found either from the Maxwellian (4.14), or directly from the kinetic equation (4.5). Still using the notation $f$ for $n F_{E, T}^{\text {iso }}$ and $\tilde{f}$ for its dimensionless version, we have

$$
j_{W}=n T^{3 / 2} \tilde{j}_{W}=W \frac{2 \sqrt{T}}{d} \tilde{j}_{W}, \quad \tilde{j}_{W}=\frac{1}{2} \int_{\mathbb{R}^{d}} \tilde{f}|\tilde{v}|^{2} \tilde{v} d \tilde{v} .
$$

Multiplying (4.8) by $|\tilde{v}|^{2}$ and integrating gives

$$
-\tilde{u}=\tilde{W} \tilde{E}-\beta \tilde{j}_{W},
$$

and therefore

$$
2 \tilde{j}_{W}=\frac{d \tilde{E}+2 \tilde{u}}{\beta}=\frac{d \beta+2}{\beta^{2}} \tilde{E} .
$$

Using (4.11), we finally obtain

$$
2 \tilde{j}_{W}=\frac{d \beta+2}{\beta+|\tilde{E}|^{2} / d} \tilde{E}
$$


This can now be expressed as a function of $\tilde{E}$ :

$$
\begin{aligned}
2 \tilde{j}_{W} & =\frac{d \sqrt{1+4|\tilde{E}|^{2} / d}+d+4}{\sqrt{1+4|\tilde{E}|^{2} / d}+1+2|\tilde{E}|^{2} / d} \tilde{E} \\
& =\frac{d \sqrt{d \nu^{2} T+4|E|^{2}}+(d+4) \sqrt{d \nu^{2} T}}{\sqrt{d \nu^{2} T+4|E|^{2}}+\left(1+2|E|^{2} /\left(d \nu^{2} T\right)\right) \sqrt{d \nu^{2} T}} \frac{E}{\nu \sqrt{T}} .
\end{aligned}
$$

As before, the last term in (4.15b) is equal to $-n E u / \epsilon$, and so we finally obtain the following system:

$$
\left\{\begin{array}{l}
\partial_{t} n+\partial_{x_{\|}}\left(n u_{\|}\right)=0 \\
\partial_{t} W+\partial_{x_{\|}}\left(W u_{W \|}\right)-\frac{1}{\epsilon} E n u=0 \\
u_{\|}=\sqrt{d T} \frac{\sqrt{d \nu^{2} T+4|E|^{2}}-\sqrt{d \nu^{2} T}}{2|E|} \\
u_{W \|}=\frac{d \sqrt{d \nu^{2} T+4|E|^{2}}+(d+4) \sqrt{d \nu^{2} T}}{d \sqrt{d \nu^{2} T+4|E|^{2}}+\left(d+2|E|^{2} /\left(\nu^{2} T\right)\right) \sqrt{d \nu^{2} T}} \frac{|E|}{\nu} \\
W=n \frac{d T}{2}
\end{array}\right.
$$

Here $x_{\|}$denotes the component of $x$ that is parallel to $E$ and $\partial_{x_{\|}}$the directional derivative in this direction; the other components act only as parameters in this problem. Back to the original physical problem, we must set $\eta=\epsilon$ in this system.

Now, the expression of the energy velocity $u_{W \|}$ can be further simplified. Introducing the auxiliary variable $z=\frac{\nu \sqrt{d T}}{2 E}$ (we assume that $E>0$ to fix the ideas), we can write

$$
\begin{aligned}
u_{\|} & =\frac{2 E}{\nu} z\left(\sqrt{z^{2}+1}-z\right), \\
u_{W \|} & =\frac{2 E}{\nu d} \frac{z\left(d \sqrt{z^{2}+1}+(d+4) z\right)}{2 z \sqrt{z^{2}+1}+2 z^{2}+1} .
\end{aligned}
$$

We first note that, upon the multiplication of $z$ by $\sqrt{d}$, the expression of $u_{\|}$is the same as in the one-dimensional case. We immediately deduce that $u_{\|}$is an increasing function of $z$ and thus of $T$. Then, by multiplying by the conjugate quantity of the denominator of (4.20), we find the simpler expression

$$
u_{W \|}=\frac{2 E}{\nu d} z\left((-d+4) z+8 z^{3}+d \sqrt{z^{2}+1}-8 z^{2} \sqrt{z^{2}+1}\right) .
$$

Returning to physical variables, this is:

$$
\begin{aligned}
u_{W \|}= & \sqrt{\frac{T}{d}} \frac{1}{(2 E)^{3}}\left[(-d+4)(2 E)^{2} \nu \sqrt{d T}+8(\nu \sqrt{d T})^{3}+\right. \\
& \left.+d(2 E)^{2} \sqrt{\nu^{2} d T+4 E^{2}}-8 \nu^{2} d T \sqrt{\nu^{2} d T+4 E^{2}}\right] .
\end{aligned}
$$

We use these expressions to show the hyperbolicity of system (4.18) below. 
4.5. Hyperbolicity of the fluid model for the isotropic thermostat. Like in the one-dimensional case (see section 3.5), we show that both $u_{\|}$and $u_{W \|}$ are nondecreasing functions of $T$ (or equivalently, of $z$ ) and that $u_{W \|}>u_{\|}$. That $u_{\|}$is nondecreasing with $z$ follows directly from the expression (4.19), which is identical to the one-dimensional expression. We now show that $u_{W \|}$ is nondecreasing with respect to $z$. We compute:

$$
\begin{aligned}
u_{W \|}^{\prime}(z) & =\frac{2 E}{\nu d} \frac{1}{\sqrt{z^{2}+1}} U(z), \\
U(z) & =32 z^{3} \sqrt{z^{2}+1}+2(-d+4) z \sqrt{z^{2}+1}-32 z^{4}+(2 d-24) z^{2}+d .
\end{aligned}
$$

We note that $U(0)=d>0$. We now show that $U(z)$ does not vanish for $z \geq 0$, which shows that $U(z) \geq 0$ for $z \geq 0$ and consequently that $u_{W \|}$ is nondecreasing. Suppose that $U(z)=0$. Then

$$
32 z^{3} \sqrt{z^{2}+1}+2(-d+4) z \sqrt{z^{2}+1}=32 z^{4}+(24-2 d) z^{2}-d .
$$

Squaring each side of this equation leads, after some algebra, to the unique root

$$
z=\frac{d}{4 \sqrt{d+4}} .
$$

Inserting this value into (4.23), we realize that this is a spurious root, introduced by the squaring procedure. Indeed, for this value of $z$, the left-hand side of (4.23) equals $\frac{2 d(d+8)}{(d+4)^{2}}$, while the right-hand side is equal to the negative of this value. Therefore, there is no positive root $z$ to the equation $U(z)=0$ which concludes the proof that $u_{W \|}$ is non decreasing with respect to $z$ and $T$.

Now, using (4.19), (4.20), we can write

$$
u_{W \|}=u_{\|}+\frac{2 E}{\nu d} 4 z^{2}\left[1-2 z\left(\sqrt{z^{2}+1}-z\right)\right] .
$$

Because $\sqrt{z^{2}+1}-z=1 /\left(\sqrt{z^{2}+1}+z\right)<1 /(2 z)$, the bracket at the right-hand side is positive and thus $u_{W \|}>u_{\|}$. This concludes the proof of the hyperbolicity of the fluid model for the isotropic thermostat.

4.6. Isotropic thermostat: remarkable limit cases and monotonicity with respect to $E$. Like in the one dimensional case, we investigate the two limit cases: $z \rightarrow \infty$ (weak field) and $z \rightarrow 0$ (strong field).

(i) Low-Field case $z \rightarrow \infty$ : then

$$
u_{\|} \sim \frac{E}{\nu}, \quad u_{W \|} \sim \frac{d+2}{d} \frac{E}{\nu} .
$$

The Low-Field mobility for the current is the same as in the one-dimensional case (see section section 3.6), and does not depend on the dimension. By contrast, the Low-Field mobility for the energy current depends on the dimension. We note that it is larger than the mobility for the current, which is consistent with the result just proven, and that, for $d=1$, we recover the value which was obtained in section 3.6.

(ii) High-Field case $z \rightarrow 0$ : then

$$
u \sim \sqrt{d T}, \quad u_{W} \sim \sqrt{d T} .
$$


Here too, the particle and energy velocities are equal and both are close to $\sqrt{d T}$. If the next order correction term is retained, for large but not infinite field values, we find

$$
u \sim \sqrt{d T}-\frac{\nu d T}{2 E}, \quad u_{W} \sim \sqrt{d T}-\left(1-\frac{4}{d}\right) \frac{\nu d T}{2 E}
$$

Again we find that $u<u_{W}$. For dimensions $d \leq 3$, as $E^{-1}$ increases, the energy velocity is larger than $\sqrt{d T}$ while the particle velocity is smaller.

In order to investigate the monotonicity with respect to $E$, we introduce the new variable $y=\frac{1}{z}=\frac{2 E}{\nu \sqrt{T}}$. In this new variable, we have

$$
\begin{aligned}
u_{\|} & =\sqrt{d T} \frac{1}{y}\left(\sqrt{1+y^{2}}-1\right), \\
u_{W \|} & =\frac{\sqrt{d T}}{d} \frac{1}{y^{3}}\left[(4-d) y^{2}+8+d y^{2} \sqrt{1+y^{2}}-8 \sqrt{1+y^{2}}\right] .
\end{aligned}
$$

The expression of $u_{\|}$as a function of $y$ is the same as for the one-dimensional model (see section 3.6) and is therefore a monotonically increasing function of $y$ and thus of $E$. At variance, the expression of $u_{W \|}$ is different and it is a surprising fact here that $u_{W \|}$ is not monotonically increasing with respect to $y$ in the physically relevant cases $d \leq 3$. By contrast, it is a monotonically increasing function of $y$ in the non-physical cases $d \geq 4$.

To prove this result, we first rewrite $(4.25)$ as

$$
u_{W \|}=\frac{d y}{\sqrt{y^{2}+1}+1}+\frac{4 y}{\left(\sqrt{y^{2}+1}+1\right)^{2}},
$$

and then compute the derivative, which after some simplification becomes

$$
u_{W \|}^{\prime}(y)=\frac{(2 d+4)\left(\sqrt{y^{2}+1}+1\right)-(4-d) y^{2}}{\left(\sqrt{y^{2}+1}+1\right)^{3} \sqrt{y^{2}+1}} .
$$

When $d \geq 4$ this obviously is strictly positive for all $y$, which implies that in this case $u_{W \|}$ is strictly increasing as a function of $y^{2}$. For $d<4$, we solve the equation $u_{W \|}^{\prime}(y)=0$, i.e.

$$
(2 d+4)\left(\sqrt{y^{2}+1}+1\right)=(4-d) y^{2} .
$$

It is easy to see that this equation has only one positive solution,

$$
y_{\max }^{2}=\frac{24(d+2)}{(4-d)^{2}} .
$$

Now, the maximal value $u_{W \|}^{\max }=u_{W \|}\left(y_{\max }\right)$ can be easily computed and leads to

$$
u_{W \|}^{\max }=\sqrt{d T} \frac{\sqrt{2}}{3 \sqrt{3}} \frac{(d+2)^{3 / 2}}{d} .
$$

The limit value of $u_{W \|}$ when $y \rightarrow \infty$ is $\sqrt{d T}$. $u_{W \|}^{\max }$ exceeds that value by the factor $\frac{\sqrt{2}}{3 \sqrt{3}} \frac{(d+2)^{3 / 2}}{d}$. This factor can be computed and is respectively equal to $\sqrt{2} \approx 1.414$, 
$\frac{4 \sqrt{2}}{3 \sqrt{3}} \approx 1.089$ and $\frac{5 \sqrt{10}}{9 \sqrt{3}} \approx 1.014$ in the cases $d=1,2$ and 3 . The corresponding values of $y_{\max }$ are $2 \sqrt{2}, 2 \sqrt{6}$ and $2 \sqrt{30}$ respectively.

Therefore, the isotropic thermostat used in conjunction with the Fokker-Planck operator leads to a remarkable negative differential velocity for the energy velocity. Usually, we find negative differential velocities in connection with collision mechanisms which have energy thresholds (such as intervalley scattering in the case of Gallium Arsenide, see e.g. [17]). Here, we find another source of negative differential velocity. Additionally, this phenomenon appears in the energy velocity but not in the particle velocity.

4.7. The leading order distribution for the parallel thermostat. In order to find the leading order distribution in the case of the parallel thermostat, we need to solve (4.7b). Similar computations as above lead to

$$
\tilde{f}(\tilde{v})=\frac{\sqrt{\beta_{\|}}}{(2 \pi)^{d / 2}} \exp \left(-\frac{\beta_{\|}}{2}\left(\tilde{v}_{\|}-\frac{|\tilde{E}|}{\beta_{\|}}\right)^{2}-\frac{\left|\tilde{v}_{\perp}\right|^{2}}{2}\right),
$$

with

$$
\beta_{\|}=1+\tilde{E} \cdot \tilde{u}=\frac{1+\sqrt{1+4|\tilde{E}|^{2}}}{2} .
$$

We note that $\beta_{\|}$satisfies the equation

$$
\beta_{\|}^{2}-\beta_{\|}-|\tilde{E}|^{2}=0
$$

which is exactly the equation for $\beta$ in the previous calculation, if one sets $d=1$.

It is then easy to check that

$$
\begin{aligned}
& \int_{\mathbb{R}^{d}} \tilde{v}_{\|} \tilde{f}(\tilde{v}) d \tilde{v}=\frac{|\tilde{E}|}{\beta_{\|}}=\tilde{u}_{\|}, \\
& \int_{\mathbb{R}^{d}} \tilde{v}_{\|}^{2} \tilde{f}(\tilde{v}) d \tilde{v}=\frac{\beta_{\|}+|\tilde{E}|^{2}}{\beta_{\|}^{2}}=1, \\
& \int_{\mathbb{R}^{d}}\left|\tilde{v}_{\perp}\right|^{2} \tilde{f}(\tilde{v}) d \tilde{v}=d-1 .
\end{aligned}
$$

Expressed in the original variables, we find that the leading order distribution function is $n F_{E, T}^{\|}$with

$$
F_{E, T}^{\|}(v)=\frac{\sqrt{\beta_{\|}}}{(2 \pi T)^{d / 2}} \exp \left(-\frac{\beta_{\|}}{2 T}\left(v_{\|}-u_{\|}\right)^{2}-\frac{\left|v_{\perp}\right|^{2}}{2 T}\right) .
$$

To conclude, the 0 -th order solution is a Gaussian, not a Maxwellian, which satisfies the property of equipartition of energy. This is achieved by a lower temperature in the direction parallel to the field.

4.8. The fluid equations for the parallel thermostat; hyperbolicity and limit cases. The strong field limit implies that both the mass flux and the energy flux are parallel to the field, and so this system is a $2 \times 2$ system, parameterized by the $x$-components orthogonal to $E$. The first order fluid equations are obtained by 
closing (4.15a), (4.15b) using the Gaussian function from above. After some easy computations, we find:

$$
\left\{\begin{array}{l}
\partial_{t} n+\partial_{x_{\|}}\left(n u_{\|}\right)=0 \\
\partial_{t} W+\partial_{x_{\|}}\left(W u_{W \|}\right)-\frac{1}{\epsilon} E n u=0 \\
u_{\|}=\frac{\sqrt{\nu^{2} T^{2}+4 T E^{2}}-\nu T}{2|E|} \\
u_{W \|}=\frac{d \sqrt{\nu^{2} T+4|E|^{2}}+(d+4) \sqrt{\nu^{2} T}}{d \sqrt{\nu^{2} T+4|E|^{2}}+d\left(1+2|E|^{2} /\left(\nu^{2} T\right)\right) \sqrt{\nu^{2} T}} \frac{|E|}{\nu} \\
W=n \frac{d T}{2}
\end{array}\right.
$$

with $\eta=\epsilon$ to match the physical setting.

It is easily shown that the change of variable $z=\frac{\nu \sqrt{T}}{2 E}$ leads to the same formulas (4.19) and (4.20) for $u_{\|}$and $u W \|$ as in the isotropic thermostat case. Therefore, formula (4.21) also holds true, and going back to physical variables, leads to

$$
\begin{aligned}
u_{W \|}= & \sqrt{\frac{T}{d}} \frac{1}{(2 E)^{3}}\left[(-d+4)(2 E)^{2} \nu \sqrt{T}+8(\nu \sqrt{T})^{3}+\right. \\
& \left.+d(2 E)^{2} \sqrt{\nu^{2} T+4 E^{2}}-8 \nu^{2} T \sqrt{\nu^{2} T+4 E^{2}}\right] .
\end{aligned}
$$

As an immediate consequence of the analysis of section 4.5 , we find that the model (4.38) is hyperbolic. We also have the following limit cases:

(i) Low-Field case $z \rightarrow \infty$ : then

$$
u_{\|} \sim \frac{E}{\nu}, \quad u_{W \|} \sim \frac{d+2}{d} \frac{E}{\nu} .
$$

(ii) High-Field case $z \rightarrow 0$ : then

$$
u \sim \sqrt{T}-\frac{\nu T}{2 E}, \quad u_{W} \sim \sqrt{T}-\left(1-\frac{4}{d}\right) \frac{\nu T}{2 E} .
$$

and the same conclusions can be drawn as in the isotropic thermostat case.

We note that the High-Field velocity is $\sqrt{T}$ instead of $\sqrt{d T}$ for the isotropic thermostat. This can be explained by the fact that, in the limit $E \rightarrow \infty$, the equilibrium distribution function for the parallel thermostat $n F_{E, T}^{\|}$has still an extension in the direction $v_{\perp}$ (in other words, is a measure supported by the hyperplane $v_{\|}=\sqrt{T}$ ), while the equilibrium distribution from two of the isotropic thermostat $n F_{E, T}^{\text {iso }}$ concentrates onto a delta distribution at the point $\sqrt{d T}$. Therefore, the parallel velocity is larger in the case of the isotropic thermostat than for the parallel thermostat. On the other hand, the Low-Field mobilities are the same.

As for the monotonicity with respect to the field, upon introducing the new variable $y=\frac{1}{z}=\frac{2 E}{\nu \sqrt{T}}, u_{\|}$and $u_{W \|}$ have the same expressions (4.24), (4.25) as in the case of the isotropic thermostat. Therefore, the same conclusions can be drawn, i.e. $u_{\|}$is a monotonically increasing function of the field but $u_{W \|}$ is first increasing towards a maximum and then decreases to its limit value $\sqrt{T}$ for $y \rightarrow \infty$. Therefore, also here, $u_{W \|}$ presents a negative differential velocity feature at High-Field. 
4.9. The High-Field limit. In this section, we investigate the High-Field limit. The fact that the particle and energy velocities saturate in this limit can be viewed as non-physical. Indeed, one could think that these velocities should become extremely large as the field becomes large. However, the fact that they saturate to the thermal velocity is paradoxically exactly what they should do. We try to explain this fact below.

We suppose that $u_{\|}$and $u_{W \|}$ are replaced by their High-Field limit $u_{\|}^{\infty}=u_{W \|}^{\infty}=$ $\sqrt{d T}$ in the case of the isotropic thermostat and $u_{\|}^{\infty}=u_{W \|}^{\infty}=\sqrt{T}$ in the case of the parallel thermostat.

For the isotropic thermostat, we find the following system (restricted to one dimension for the sake of convenience):

$$
\left\{\begin{array}{l}
\partial_{t} n+\partial_{x}\left(n u^{\infty}\right)=0 \\
\partial_{t} W+\partial_{x}\left(W u^{\infty}\right)-\frac{1}{\eta} E n u^{\infty}=0 \\
u^{\infty}=\sqrt{d T} \operatorname{Sign}(E) .
\end{array}\right.
$$

Here we take into account that $E$ can be positive or negative and we have to make sure that $u^{\infty}$ points in the same direction as $E$.

The corresponding High-Field drift-diffusion system (i.e. when only the continuity equation is retained) coupled with the Poisson equation for the field has been studied in [32] where existence of solutions is proved by a regularization procedure and numerical simulations are given. The study of the present more general system is in progress.

Here, we focus on the very simple case where $E$ has a constant sign. Then, we can recast the second equation (4.40) into an equation for the velocity $u^{\infty}$ itself which we simply denote by $u$. We indeed find that the system is equivalent to the Pressureless Gas Dynamics equations with a force field:

$$
\left\{\begin{array}{l}
\partial_{t} n+\partial_{x}(n u)=0 \\
\partial_{t} u+u \partial_{x} u=\frac{1}{\eta} E
\end{array}\right.
$$

This system is actually the system of equations that ballistic particles would follow. Indeed, taking the collisionless Vlasov equation (i.e. (4.4) without the collision and thermostat terms) and looking for ballistic solutions of the form of delta measure solutions $f(x, v, t)=n(x, t) \delta(v-u(x, t))$ would exactly lead to system (4.41) for $n$ and $u$. Therefore, the high field velocities $u_{\|}^{\infty}=u_{W \|}^{\infty}=\sqrt{d T}$ have exactly the right value which allows us to recover a ballistic particle, which is how they should behave. We refer to [8], [9], [31] for references on Pressureless Gas Dynamics.

Note that in the case of the parallel thermostat, because the limiting velocity is $\sqrt{T}$ instead of $\sqrt{d T}$, the corresponding velocity equation becomes

$$
\partial_{t} u+u \partial_{x} u=\frac{1}{d \eta} E
$$

This equation corresponds to a ballistic particle in a field divided by $d$ and is not the correct ballistic limit. This is an argument for preferring the isotropic thermostat rather than the parallel one. 


\section{Conclusion and future developments}

In this paper, we have proposed a novel approach for deriving mass and energy balance laws for dissipative systems subject to a large driving force. This approach consists in using a thermostat which makes the composite operator constructed on the field and collision operators globally mass and energy-conservative. We have restricted ourselves to the leading order in the Hilbert expansion. The resulting models are hyperbolic systems of balance laws for the local density and energy. This approach has been carried out in the framework of two simplified collision operators: a one-dimensional BGK operator and a multi-dimensional Fokker-Planck operator.

Clearly, in order to be able to produce physically realistic models, this approach should be continued to include second order diffusive corrections as would be obtained by a Chapman-Enskog procedure. Without such diffusive corrections, the model does not retain enough physical detail. After these diffusive corrections are introduced, the model will be ready for numerical simulations. This program will be carried out in future work.

Another path towards more physically realistic models is the inclusion of more realistic collision operators, such as impurity and phonon scattering in the case of semiconductors, and scattering against ions and neutral molecules in the case of plasmas. If a complex collision operator is used, the simple algebraic manipulations which allowed us to get analytical expressions of the High-Field equilibrium distribution functions and of the velocity-field or temperature relationships would no lower be possible. One should resort to more analytic techniques to prove the existence of such equilibria, and to numerical simulations to compute the velocity functions. This is another track for current research.

Finally, the mathematical analysis of the resulting systems (in particular when the field is coupled with the particles through Maxwell's or Poisson's equations) is another open question. The special case of the High-Field limit as expounded in section 4.9 is of particular interest as it provides a very simple model with potentially novel mathematical features.

\section{REFERENCES}

[1] Y. Apanovich, E. Lyumkis, B. Polsky, A. Shur and P. Blakey, Steady-state and transient analysis of submicron devices using energy balance and simplified hydrodynamic models, I. E. E. E. Trans. CAD of Integ. Circuits and Systems, 13, 702-711, 1994.

[2] N. Ben Abdallah and P. Degond, On a hierarchy of macroscopic models for semiconductors, J. Math. Phys., 37, 3306-3333, 1996.

[3] N. Ben Abdallah, P. Degond and S. Gnieys, An energy-transport model for semiconductors derived from the Boltzmann equation, J. Stat. Phys., 84, 205-231, 1996.

[4] N. Ben Abdallah, P. Degond, P. Markowich and C. Schmeiser, High-field approximations of the spherical harmonics expansion model for semiconductors, Zeitschrifts für Angewandte Mathematik und Physik, 52, 201-230, 2001.

[5] V. Bagland, B. Wennberg and Y. Wondmagegne, Stationary states for the non-cutoff Kac equation with a Gaussian thermostat, preprint, 2005.

[6] F. Bonetto, D. Daems and J. L. Lebowitz, Properties of stationary nonequilibrium states in the thermostatted periodic Lorentz gas I: the one particle system, J. Statist. Phys., 101, 35-60, 2000.

[7] F. Bonetto, D. Daems, J. L. Lebowitz and V. Ricci, Properties of stationary nonequilibrium states in the thermostatted periodic Lorentz gas: the multiparticle system, Phys. Rev. E (3), 65, 051-204, 2002.

[8] F. Bouchut, On zero pressure gas dynamics, in Advances in kinetic theory and computing, B. Perthame (ed), World Scientific, 171-190, 1994.

[9] F. Bouchut and F. James, Duality solutions for pressureless gases, monotone scalar conser- 
vation laws, and uniqueness, Comm. Partial Differential Equations, 24(11-12), 2173-2189, 1999.

[10] G. Busoni, Giorgio and G. Frosali, Asymptotic scattering operators and drift velocity in charged particle transport problems, J. Math. Phys., 34, 4668-4691, 1993.

[11] G. Busoni, Giorgio and G. Frosali, On the long-time behaviour of runaway solutions in charged particle transport, Transport Theory Stat. Phys., 25, 353-367, 1996.

[12] C. Cercignani, I. Gamba and D. Levermore, A high field approximation to a Boltzmann-Poisson system in bounded domains, Appl. Math. Lett., 4, 111-118, 1997.

[13] S. Chapman, The kinetic theory of simple and composite gases: viscosity, thermal conduction and diffusion, Proc. Roy. Soc. (London), A93, 1-20, 1916/17.

[14] D. Chen, E. Kan, U. Ravaioli, C. Shu and R. Dutton, An improved energy transport model including nonparabolicity and non-Maxwellian distribution effects, IEEE Electr. Dev. Letters, 13, 26-28, 1992

[15] N. I. Chernov, G. L. Eyink, J. L. Lebowitz and Ya. G. Sinai, Derivation of Ohm's law in a deterministic mechanical model, Phys. Rev. Lett., 70, 15, 1993.

[16] N. I. Chernov, G. L. Eyink, J. L. Lebowitz and Ya. G. Sinai, Steady-state electrical conduction in the periodic Lorentz gas, Comm. Math. Phys., 154, 569-601, 1993.

[17] E. M. Conwell, High-Field Transport in Semiconductors, Solid State Physics Vol. g, Academic Press, New York, 1967.

[18] P. Degond, Mathematical modelling of microelectronics semiconductor devices, Proceedings of the Morningside Mathematical Center, Beijing, AMS/IP Studies in Advanced Mathematics, AMS Society and International Press, 77-109, 2000.

[19] P. Degond, Asymptotic continuum models for plasmas and disparate mass gaseous binary mixtures, paratre dans 'Material substructures in complex bodies: from atomic level to continuum', G. Capriz, P-M. Mariano (eds), Birkhauser.

[20] P. Degond, S. Gnieys et A. Jüngel, A system of parabolic equations in nonequilibrium thermodynamics including thermal and electrical effects, J. Math. Pures Appl., 76, 991-1015, 1997.

[21] P. Degond, A. Jüngel and P. Pietra, Numerical discretization of energy-transport models for semiconductors with non-parabolic band structure, SIAM Sci. Comp., 22, 986-1007, 2000.

[22] P. Degond and A. Jüngel, High field approximations of the energy-transport model for semiconductors with non-parabolic band structure, Zeitschrifts für Angewandte Mathematik und Physik, 52, 1053-1070, 2001.

[23] P. Degond and B. Lucquin-Desreux, Transport coefficients of plasmas and disparate mass binary gases, Transp. Theory Stat. Phys., 25, 595-633, 1996.

[24] D. Enskog, Kinetische Theorie der Vorgänge in mässig verdünntent Gasen, 1, Allgemeiner Teil, Almqvist Wiksell, Uppsala, 1917.

[25] D. J. Evans, Computer experiment for non-linear thermodynamics of couette-flow, J. Chem. Phys., 78, 3297-3302, 1983.

[26] D. J. Evans, W. G. Hoover, B. H. Failor, B. Moran and A. J. C. Ladd, Non-equilibrium molecular-dynamics via Gauss principle of least constraint, Phys. Rev. A, 8, 1016-1021, 1983.

[27] D. J. Evans and B. L. Holian, The Nose-Hoover thermostat, J. Chem. Phys., 83(8), 4069-4074, 1985.

[28] D. J. Evans and G. P. Morriss, Statistical Mechanics of Nonequilibrium Liquids, Academic press, London, 1990.

[29] G. Frosali and C. van der Mee, Asymptotic behaviour of drift velocity with spatial diffusion of electrons, Transport Theory Stat. Phys., 24, 241-26, 1952.

[30] F. Golse and F. Poupaud, Limite fluide des equations de Boltzmann des semiconducteurs pour une statistique de Fermi-Dirac, Asympt. Analysis, 6, 135-160, 1992.

[31] E. Grenier, Existence globale pour le système des gaz sans pression, C. R. Acad. Sci. Paris Sér. I Math., 321(2), 171-174, 1995.

[32] J. Haskovec and C. Schmeiser, Transport in semiconductors at saturated velocities, Comm. Math. Sci., 3, 219-233, 2005.

[33] D. Hilbert, Begründung der kinetischen Gastheorie, Math. Ann., 72, 562-577, 1916/17.

[34] S. Holst, A. Jngel and P. Pietra, An adaptive mixed scheme for energy-transport simulations of field-effect transistors, SIAM J. Sci. Comp., 25, 1698-1716, 2004.

[35] W. G. Hoover, A. J. C. Ladd and B. Moran, High-strain-rate plastic flow studied via nonequilibrium molecular dynamics, Phys. Rev. Lett., 48, 1818-1820, 1982.

[36] W. G. Hoover, Molecular dynamics, Lect. Notes Phys., Springer Verlag, 258, 1986.

[37] W. G. Hoover, Computational Statistical Mechanics, Elsevier, Amsterdam, 1991.

[38] W. G. Hoover, K. Aoki, C. G. Hoover and S. V. DeGroot, Time-reversible deterministic ther- 
mostats, Physica D, 187, 253-267, 2004.

[39] M. Kac, Foundations of kinetic theory, Proceedings of the Third Berkeley Symposium on Mathematical Statistics and Probability, University of California Press, III, 171-197, 19541955, Berkeley and Los Angeles, 1956.

[40] E. Lyumkis, B. Polsky, A. Shur and P. Visocky, Transient semiconductor device simulation including energy balance equation, Compel, 11, 311-325, 1992.

[41] P. A. Markowich, C. Ringhofer and C. Schmeiser, Semiconductor Equations, Springer Wien, 1990.

[42] G. P. Morriss, C. P. Dettmann, Thermostats: analysis and application, Chaos, 8(2), 321-336, 1998.

[43] S. Nose, A molecular dynamics method for simulations in the canonical ensemble, Molecular Phys., 52(2), 255-268, 1984.

[44] F. Poupaud, Diffusion approximation of the linear semiconductor equation: analysis of boundary layers, Asymp. Anal., 4, 293-317, 1991.

[45] F. Poupaud, Critres d'existence de solutions stationnaires homognes en thorie cintique des semi-conducteurs. Application l'approximation fluide champ fort, C. R. Acad. Sci. Paris Sr. I Math., 308, 381-386, 1989.

[46] F. Poupaud, Derivation of a hydrodynamic system hierarchy for semiconductors from the Boltzmann equation, Appl. Math. Lett., 4, 1, 75-79, 1991.

[47] F. Poupaud, Runaway phenomena and fluid approximation under high fields in semiconductor kinetic theory, Z. Angew. Math. Mech., 72, 359-372, 1992.

[48] K. Souissi, F. Odeh, H. H. K. Tang and A. Gnudi, Comparative studies of hydrodynamic and energy-transport models, Compel, 13, 439-453, 1994.

[49] R. Stratton, Diffusion of hot and cold electrons in semiconductor barriers, Phys. Rev., 126, 2002-2014, 1962.

[50] W. V. Van Roosebroeck, Theory of flow of electrons and holes in germanium and other semiconductors, Bell Syst. Tech. J., 29, 560-607, 1950.

[51] B. Wennberg and Y. Wondmagegne, The Kac equation with a thermostatted force field, J. Statist Phys., 124(2-4), 859-880, 2006.

[52] B. Wennberg and Y. Wondmagegne, Stationary states for the Kac equation with a Gaussian thermostat, Nonlinearity, 17, 633-648, 2004.

[53] Y. Wondmagegne, Kinetic equations with a Gaussian thermostat, Doctoral thesis, Department of Mathematical Sciences, Chalmers University of Technology and Göteborg University, Göteborg, 2005. 